Prepared for the U.S. Department of Energy

under Contract DE-AC05-76RL01830

\title{
Engineering Evaluation of X/Q Values Consistent with Regulatory Guide 1.145
}

S. B. Ross
J. P. Rishel
P. P. Lowry

February, 2010

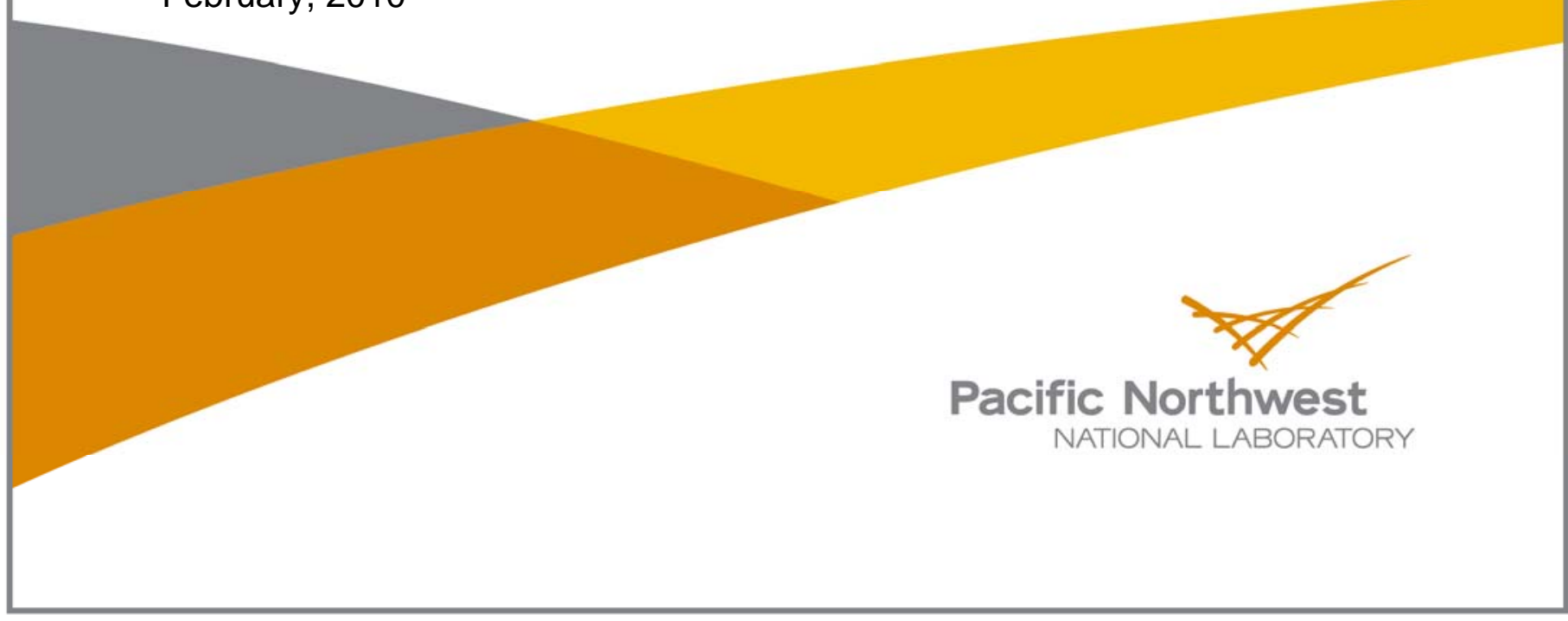




\title{
DISCLAIMER
}

This report was prepared as an account of work sponsored by an agency of the United States Government. Neither the United States Government nor any agency thereof, nor Battelle Memorial Institute, nor any of their employees, makes any warranty, express or implied, or assumes any legal liability or responsibility for the accuracy, completeness, or usefulness of any information, apparatus, product, or process disclosed, or represents that its use would not infringe privately owned rights. Reference herein to any specific commercial product, process, or service by trade name, trademark, manufacturer, or otherwise does not necessarily constitute or imply its endorsement, recommendation, or favoring by the United States Government or any agency thereof, or Battelle Memorial Institute. The views and opinions of authors expressed herein do not necessarily state or reflect those of the United States Government or any agency thereof.

\author{
PACIFIC NORTHWEST NATIONAL LABORATORY \\ operated by \\ BATTELLE \\ for the \\ UNITED STATES DEPARTMENT OF ENERGY \\ under Contract DE-AC05-76RL01830
}

Printed in the United States of America
Available to DOE and DOE contractors from the
Office of Scientific and Technical Information,
P.O. Box 62, Oak Ridge, TN 37831-0062;
ph: (865) 576-8401
fax: (865) 576-5728
email: reports@adonis.osti.gov

\author{
Available to the public from the National Technical Information Service, \\ U.S. Department of Commerce, 5285 Port Royal Rd., Springfield, VA 22161 \\ ph: (800) $553-6847$$$
\text { fax: (703) 605-6900 }
$$ \\ email: orders@ntis.fedworld.gov \\ online ordering: http://www.ntis.gov/ordering.htm
}

This document was printed on recycled paper.

$(9 / 2003)$ 


\section{Engineering Evaluation of X/Q Values Consistent with Regulatory Guide 1.145}

S. B. Ross

J.P. Rishel

P. P. Lowry

February, 2010

Prepared for

the U.S. Department of Energy

under Contract DE-AC05-76RL01830

Pacific Northwest National Laboratory

Richland, Washington 99352 



\section{CONTENTS}

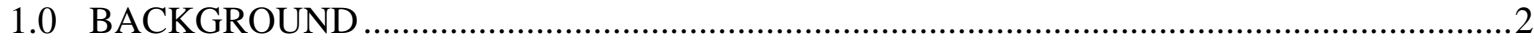

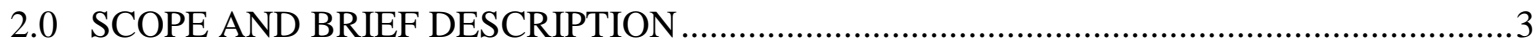

3.0 DESIGN OR TECHNICAL PARAMETER INPUT AND SOURCES ..................................

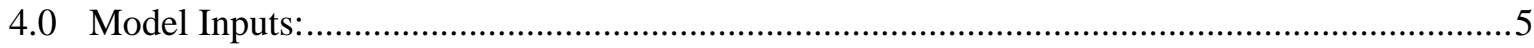

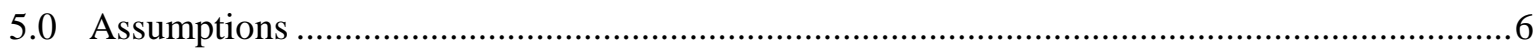

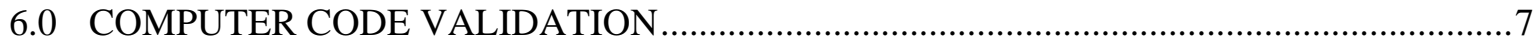

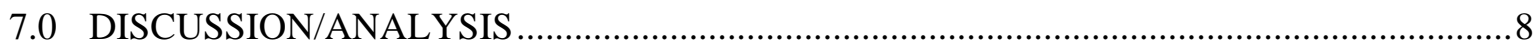

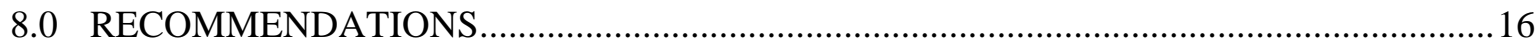

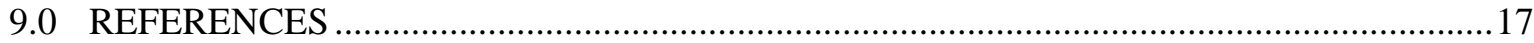

\section{APPENDIXES}

Appendix A - PAVAN Input/Output Files (Provided Electronically) ….......................................19

Appendix B - Appendix B QA Record …….......................................................................20 
PNNL-19217

\subsection{BACKGROUND}

The Next Generation Nuclear Plant (NGNP) will be a demonstration of the technical, licensing, operational, and commercial viability of High Temperature Gas-Cooled Reactor (HTGR) technology for the production of process heat, electricity, and hydrogen. This nuclear based technology can provide high-temperature process heat that can be used as a substitute for the burning of fossil fuels for a wide range of commercial applications. The substitution of the HTGR for burning fossil fuels conserves these hydrocarbon resources for other uses, reduces uncertainty in the cost and supply of natural gas and oil, and eliminates the emissions of greenhouse gases attendant with the burning of these fuels.

In the Energy Policy Act of 2005 (EPAct), the Department of Energy (DOE) was tasked with providing a demonstration of this HTGR technology to economically and reliably produce electricity and hydrogen by the year 2021. As the lead nuclear technology development laboratory of the DOE, the Idaho National Laboratory (INL) has initiated the work necessary to complete this task. The results and conclusions of this work define key technical risks and specific requirements that will govern the evaluations and design development required to be completed in continuing NGNP design progression.

This report aids in advancing the design progression of the NGNP, providing bases for interfacing with potential end-users and for obtaining initial input from the NRC to allow future design activities to proceed with regulatory certainty. Final decisions on the operating conditions and configuration of the NGNP including site specific atmospheric dispersion estimates will be established during subsequent design development.

In this evaluation, PAVAN was used to calculate X/Q values at defined downwind distances from hypothetical ground-level and elevated releases using meteorological data from 18 nuclear sites 


\subsection{SCOPE AND BRIEF DESCRIPTION}

Regulatory Guide (RG) 1.145 (NRC 1983) provides guidance on calculating atmospheric dispersion (X/Q) estimates for the assessment of the consequences of design basis accidents for nuclear power stations. Such assessments are required under 10 CFR 100 and 10 CFR 52. PAVAN (NUREG/CR-2858) is an atmospheric dispersion model that implements the guidance contained in RG 1.145 (NRC 1983). PAVAN uses joint frequency distributions (JFDs) of wind direction, wind speed, and atmospheric stability class to estimate X/Q values for specific averaging time periods at specified distances. The model is based on a straight-line Gaussian model that assumes the release rate is constant for the entire period of the release. 


\subsection{DESIGN OR TECHNICAL PARAMETER INPUT AND SOURCES}

In this evaluation, PAVAN was used to calculate X/Q values at defined downwind distances from hypothetical ground-level and elevated releases using meteorological data from 18 nuclear sites identified in Table 3.1. Since the model inputs (i.e., release heights, boundary distances) used in this evaluation are hypothetical, the sites are identified using alpha characters so as to not confuse this evaluation as an actual analysis for any particular site. The sites are ordered with respect to increasing mean wind speed and cover a range of meteorological conditions. In total, 73 years of meteorological data were used in the analysis.

Table 3.1: Sites with meteorological data evaluated in PAVAN

$\begin{array}{cccccc}\text { Site ID } & \begin{array}{c}\text { Characterization } \\ \text { Chan }\end{array} & \begin{array}{c}\text { Mean Wind } \\ \text { Speed (m/s) }\end{array} & \begin{array}{c}\text { Calms (\%) } \\ \text { Aissing (\%) }\end{array} & \begin{array}{c}\text { Years } \\ \text { Evaluated }\end{array} \\ \text { B } & \text { Inland } & 1.55 & 0.51 & 0.70 & 5 \\ \text { C } & \text { Inland } & 1.64 & 0.98 & 1.26 & 5 \\ \text { D } & \text { Inland } & 1.66 & 6.13 & 5.61 & 5 \\ \text { E } & \text { Inland } & 1.83 & 0.45 & 4.10 & 2 \\ \text { F } & \text { Inland } & 2.05 & 1.27 & 2.32 & 5 \\ \text { G } & \text { Inland } & 2.07 & 0.06 & 6.32 & 5 \\ \text { H } & \text { Inland } & 2.14 & 0.50 & 0.92 & 6 \\ \text { I } & \text { Inland } & 2.17 & 0.14 & 3.06 & 5 \\ \text { J } & \text { Inland } & 2.25 & 0.13 & 0.81 & 1 \\ \text { K } & \text { Inland } & 2.39 & 0.44 & 3.77 & 5 \\ \text { L } & \text { Inland } & 2.43 & 0.05 & 0.83 & 3 \\ \text { M } & \text { Inland } & 2.79 & 4.91 & 4.11 & 2 \\ \text { N } & \text { Coastal } & 2.85 & 0.95 & 2.09 & 5 \\ \text { O } & \text { Coastal } & 3.36 & 0.55 & 5.86 & 5 \\ \text { P } & \text { Coastal } & 3.38 & 0.06 & 0.25 & 4 \\ \text { Q } & \text { Inland } & 3.67 & 0.10 & 6.30 & 3 \\ \text { R } & \text { Coastal } & 4.06 & 0.00 & 3.31 & 2 \\ & \text { Inland } & 4.40 & 3.37 & 6.67 & 5\end{array}$

Both a ground-level and elevated release are simulated in PAVAN to provide bounds on the estimates of X/Q at defined downwind distances. Ground-level releases result in maximum $X / Q$ 's near the release point that decrease monotonically as a function of downwind distance. Elevated releases result in X/Q values that initially increase with downwind distance from the release point, reach a maximum, and then decrease thereafter. For an elevated release, the PAVAN model automatically determines if the maximum X/Q is beyond the boundary; if so, the model conservatively uses the maximum value as the boundary value. 
PNNL-19217

\subsection{Model Inputs:}

PAVAN has basic model input requirements, including release type and height, building dimensions to calculate wake effects, and downwind $X / Q$ evaluation distances (e.g., the EAB or LPZ sector-dependent distances). For this evaluation, both a ground-level and elevated release were used to estimate X/Q at a range of downwind distances from the release, including 400, $800,1600,3200,8000$ meters. Because PAVAN has no provision for calculating an effective plume height from buoyancy or mechanical jet effects, an elevated release height of 100 meters was selected. Releases that might occur between ground-level and 100 meters are therefore expected to be bounded by these results. Actual release heights can be calculated using the method of Briggs (1969) when effluent temperature and exit velocity are known, as suggested in RG 1.145 (NRC 1983) and RG 1.111 (NRC 1977).

PAVAN requires meteorological data in the form of a joint frequency distribution (JFD) of wind direction, wind speed, and atmospheric stability. The JFD's summarize wind information using 16 sectors based on standard cardinal compass directions (i.e., N, NNE, etc.) and wind speed bins defined in RG 1.23 Revision 1 (NRC 2007). Vertical temperature difference is used to estimate the Pasquill stability classes (NRC 2007). The hourly data were received as part of license renewals or along with Environmental Reports (ER's) for Early Site Permit (ESP)/Combined Licensing (COL) license applications from 18 sites identified in Table 1.1. As part of developing PAVAN08 (commissioned by NRC to update/replace the original version of PAVAN (NUREG/CR-2858), a subroutine was developed which writes the original PAVAN input file, including the JFD from the hourly data provided by the utilities. This set of data and the PAVAN output is being used to generically to develop an envelope of X/Q's for existing reactor sites. 
PNNL-19217

\subsection{Assumptions}

Building wake-effects were not included, since the building wake acts to increase diffusion and lower the X/Q's, especially close to the release point, this assumption is conservative. The release information used in this evaluation is summarized in Table 5.1.

Table 5.1: Release information used in PAVAN simulations.

\begin{tabular}{|l|l|}
\hline \multicolumn{2}{|l|}{ Release Characteristics: } \\
\hline Release Type & Ground-level and Elevated $(100 \mathrm{~m})$ \\
\hline Terrain & Flat \\
\hline Building Wake & No \\
\hline Evaluation & $400,800,1600,3200,8000$ \\
Distances (m) & \\
\hline
\end{tabular}


PNNL-19217

\subsection{COMPUTER CODE VALIDATION}

a. $\quad$ Computer type: Intel Xeon CPU 2.33 Ghz running Windows XP Service Pack 3

b. Computer program name and revision: PAVAN, 10/76, 8/79 REVISION, IMPLEMENTATION OF REGULATORY GUIDE 1.145

c. Inputs: See Appendix A

d. Outputs: See Appendix A

e. $\quad$ Evidence of, or reference to, computer program validation: Test case input files were run and output was validated against test case output files.

f. Bases supporting application of the computer program to the specific physical problem: PAVAN, which is a program used by the U.S. Nuclear Regulatory Commission to estimate downwind ground-level air concentrations for potential accidental releases of radioactive material from nuclear facilities. Such an assessment is required by 10 CFR Part 100 and 10 CFR Part 50. The program implements the guidance provided in Regulatory Guide -1.145, "Atmospheric Dispersion Models for Potential Accident Consequence Assessments at Nuclear Power Plants." PAVAN (An Atmospheric Dispersion Program for Evaluating Design Basis Accidental Releases of Radioactive Materials from Nuclear Power Stations) was written to implement the guidance in Regulatory Guide 1.145 (Atmospheric Dispersion Models for Evaluating Design Basis Accidents at Nuclear Power Plants). 


\subsection{DISCUSSIONIANALYSIS}

PAVAN was run for both a ground-level and elevated (100-meter) release using meteorological data from 18 sites identified in Table 1.1. Estimates of X/Q were made at various distances downwind of the release, including 400, 800, 1600, 3200, 8000 meters.

Following the guidance of RG 1.145 (NRC 1983), PAVAN calculates X/Q at various percentiles for the 0-2 hour averaging period as well as an overall site annual average. The $50^{\text {th }}$ and $95^{\text {th }}$ percentiles form the basis of environmental and safety evaluations, respectively.

A goal for the NGNP is to demonstrate compliance with regulatory dose limits bases on an exclusion area boundary and emergency planning zones boundaries set nominally to 400 meters. This paper presents the development of the atmospheric dispersion (X/Q) estimates for use in calculating doses at this distance and evaluating the resultant X/Q for an 800 meter receptor against X/Q values submitted to the NRC for review as part of Design Control Documentation (DCD) in support of future COL Applications.

For this evaluation, PAVAN was used to calculate X/Q values at defined downwind distances from hypothetical ground-level and elevated releases using meteorological data from 18 nuclear sites with varying meteorological characteristics. X/Q values where calculated for distances of $400,800,1600,3200$, and 8000 meters. PAVAN (NUREG/CR-2858) is an atmospheric dispersion model that implements the guidance contained in RG 1.145 (NRC 1983). PAVAN uses joint frequency distributions (JFDs) of wind direction, wind speed, and atmospheric stability class to estimate $\mathrm{X} / \mathrm{Q}$ values for specific averaging time periods at specified distances.

The Maximum X/Q values for 400 and 800 meters determined from this evaluation are presented in Table 7.1.

TABLE 7.1 PAVAN Ground Level Release X/Q values calculated per RG 1.145.

\begin{tabular}{|l|c|}
\hline \multicolumn{1}{|c|}{ Distance Evaluated } & $\begin{array}{c}\text { Maximum } \\
\text { Calculated } \\
\text { X/Q }\end{array}$ \\
\hline EAB -400 meters (0-2 hrs) 95\% & $3.35 E-03$ \\
\hline 800 meters (0-2 hrs) 95\% & $1.05 E-03$ \\
\hline EAB-400 meters annual average & $1.60 E-04$ \\
\hline 800 meters annual average & $4.63 E-05$ \\
\hline
\end{tabular}

The maximum ground-level X/Q values (typically at 0.5 miles) contained within DCD currently approved or undergoing review for the Generation III LWRs are presented in Table 7.2. 
TABLE 7.2 X/Q values from submittals to NRC.

\begin{tabular}{|l|l|c|c|l|}
\hline Reactor & \multicolumn{1}{|c|}{$\begin{array}{c}\text { EAB } \\
\text { distance }\end{array}$} & $\begin{array}{c}\mathbf{( 0 - 2} \mathbf{~ h r ) ~ X / Q} \\
\mathbf{s} / \mathbf{m}^{\mathbf{3}}\end{array}$ & $\begin{array}{c}\text { Annual X/Q } \\
\mathbf{s}^{\mathbf{3}} \mathbf{3}\end{array}$ & \multicolumn{1}{c|}{ Reference } \\
\hline APWR & $0.5 \mathrm{mi}$ & $5 \mathrm{E}-04$ & $1.6 \mathrm{E}-05$ & $\begin{array}{l}\text { Table 2.0-1 Rev 1 } \\
\text { ADAMS Accession\# ML082480583 }\end{array}$ \\
\hline U.S. EPR & $0.5 \mathrm{mi}$ & $1 \mathrm{E}-03$ & $4.973 \mathrm{E}-06$ & $\begin{array}{l}\text { Table 2.1-1 Rev 0 } \\
\text { ADAMS Accession \# ML073531435 }\end{array}$ \\
\hline AP1000 & $0.5 \mathrm{mi}$ & $5.1 \mathrm{E}-04$ & $2 \mathrm{E}-05$ & $\begin{array}{l}\text { Table 2-1 Rev 17 } \\
\text { ADAMS Accession \# ML083230296 }\end{array}$ \\
\hline ABWR & $0.5 \mathrm{mi}$ & $1.37 \mathrm{E}-3$ & & $\begin{array}{l}\text { Table 2.0-1, Rev. 0 } \\
\text { ABWR DCD Tier 2 Chapter 2 } \\
\text { (Table 2-2 NUREG-1503) }\end{array}$ \\
\hline ESBWR & & $2.00 \mathrm{E}-03$ & & $\begin{array}{l}\text { Table 2.0-1, Rev. 05 } \\
\text { ADAMS Accession\# ML081820391 }\end{array}$ \\
\hline
\end{tabular}

As shown in Table 7.3 the calculated maximum 0-2 hours X/Q value at 800 meters (1.051E-03) corresponds to the values contained in Table 7.1.

The calculated maximum average annual X/Q values at 800 meters (4.63E-05) also corresponds well and bounds the values contained within the DCDs.

TABLE 7.3 Comparison of XIQ values.

\begin{tabular}{|l|c|c|}
\hline Reactor & $\begin{array}{c}\mathbf{( 0 - 2} \mathbf{~ h r}) \mathbf{X} / \mathbf{Q} \\
\mathbf{s} / \mathbf{m} 3\end{array}$ & $\begin{array}{c}\text { Annual X/Q } \\
\mathbf{s} / \mathbf{m} 3\end{array}$ \\
\hline APWR & $5 \mathrm{E}-04$ & $1.6 \mathrm{E}-05$ \\
\hline AP1000 & $5.1 \mathrm{E}-04$ & $2 \mathrm{E}-05$ \\
\hline U.S. EPR & $1 \mathrm{E}-03$ & $4.973 \mathrm{E}-06$ \\
\hline PAVAN 800 meters (0-2 hrs) 95\% & $\mathbf{1 . 0 5 E - 0 3}$ & $\mathbf{4 . 6 3 E - 0 5}$ \\
\hline ABWR & $1.37 \mathrm{E}-3$ & \\
\hline ESBWR & $2.00 \mathrm{E}-03$ & \\
\hline
\end{tabular}

Based on this evaluation, the use of the $95 \%$ X/Q values presented in this calculation and shown in Table 7.4 for the 400 meter distance appear reasonable for use for the NGNP scoping calculations for comparison against acceptance criteria for the EAB and LPZ boundaries. The use of the elevated (100 meter) release height X/Q values could be considered for large depressurized loss of forced cooling (DLOFC) events assuming venting of hot gases to the environment, however additional work should be performed to benchmark and incorporate a buoyant gas model into the code. 
TABLE $7.495 \%$ X/Q values

\begin{tabular}{|c|c|c|c|c|c|c|}
\hline \multirow{2}{*}{$\begin{array}{c}\text { Release } \\
\text { Assumption }\end{array}$} & \multicolumn{6}{|c|}{ Time Interval } \\
\cline { 2 - 7 } & $0-2$ hour & $0-8 \mathrm{hr}$ & $8-24 \mathrm{hr}$ & $1-4 d a y$ & $4-30$ day & Annual \\
\hline $\begin{array}{c}\text { 95\% Ground } \\
\text { level }\end{array}$ & $3.35 \mathrm{E}-03$ & $1.91 \mathrm{E}-03$ & $1.49 \mathrm{E}-03$ & $8.75 \mathrm{E}-04$ & $4.08 \mathrm{E}-04$ & $1.60 \mathrm{E}-04$ \\
\hline $\begin{array}{c}\text { 95\% Elevated } \\
\text { (buoyant gas) }\end{array}$ & $6.17 \mathrm{E}-06$ & $3.33 \mathrm{E}-06^{1}$ & $2.54 \mathrm{E}-06^{1}$ & $1.41 \mathrm{E}-06^{1}$ & $6.08 \mathrm{E}-07^{1}$ & $2.40{\mathrm{E}-07^{1}}^{1}$ \\
\hline
\end{tabular}

1 Use of elevated release height for release durations greater than $2 \mathrm{hrs}$ may not be appropriate unless a stack release point is verified.

Tables 7.5 through 7.7 summarize X/Q's for the $0-2$ hour $50^{\text {th }}$ percentile, $0-2$ hour $95^{\text {th }}$ percentile, and annual average for a ground-level release. Similarly, Tables 7.8 through 7.10 summarize X/Q's for the $0-2$ hour $50^{\text {th }}$ percentile, 0-2 hour $95^{\text {th }}$ percentile, and annual average for an elevated (100-meter) release. For ground-level and elevated releases, the 95 percentile values were the values that were exceeded 5 percent of the time. For ground-level releases, the 5 percent overall site summary tables in the PAVAN output provided the necessary data. For elevated releases, the 5 percent overall site summary tables were not created by PAVAN and the detailed 5 percent overall site results in the PAVAN output were used. Because the summary tables were not created, the X/Q values did not correspond to exactly 50 or 95 percent, and were instead were the $X / Q$ values that corresponded to the largest probabilities less than or equal to 50 or 95 percent.

PAVAN does not explicitly calculate X/Q's for intermediate (i.e., 0-8 hour, 8-24 hour, 1-4 day, and 4-30 day) averaging times. However, the values can be approximated by using the methodology of RG 1.145 (NRC 1983), which involves logarithmic interpolation between the 0-2 hour and annual average. Tables 7.11 and Table 7.12 summarize the ground-level $400-\mathrm{m}$ X/Q's for the intermediate averaging times for the $50^{\text {th }}$ and $95^{\text {th }}$ percentile, respectively. Tables 7.13 and 1.14 are identical tables, except for the elevated release. Similar tables can easily be constructed for the other downwind distances (i.e., 800, 1600, 3200, and 8000 meter) using logarithmic interpolation between the 0-2 hour and annual average values. For calculations using realistic estimates of dispersion parameters, (e.g., Environmental Report, risk evaluations) the $50 \%$ X/Q values are presented in Table 7.5 for the identified distances from 400 to 8000 meters. 
Table 7.5: Ground-level $50^{\text {th }}$ Percentile 0-2 hour Average X/Q $\left(\mathrm{s} / \mathrm{m}^{3}\right)$.

\begin{tabular}{|c|c|c|c|c|c|}
\hline Site ID & $400 \mathrm{~m}$ & $800 \mathrm{~m}$ & $1600 \mathrm{~m}$ & $3200 \mathrm{~m}$ & $8000 \mathrm{~m}$ \\
\hline A & 2.859E-04 & 9.076E-05 & 3.814E-05 & 1.622E-05 & 5.562E-06 \\
\hline B & 2.891E-04 & 9.072E-05 & 4.459E-05 & 1.851E-05 & 6.330E-06 \\
\hline C & 2.845E-04 & 9.010E-05 & 3.924E-05 & 1.703E-05 & 5.263E-06 \\
\hline D & $2.535 \mathrm{E}-04$ & 8.002E-05 & 3.411E-05 & 1.436E-05 & 4.418E-06 \\
\hline E & 2.349E-04 & 7.436E-05 & 2.929E-05 & 1.205E-05 & 3.736E-06 \\
\hline F & 2.110E-04 & 6.674E-05 & 2.750E-05 & 1.127E-05 & 3.319E-06 \\
\hline G & 2.423E-04 & 7.702E-05 & 3.167E-05 & 1.283E-05 & $4.026 \mathrm{E}-0$ \\
\hline $\mathrm{H}$ & 2.373E-04 & 7.477E-05 & 3.137E-05 & 1.279E-05 & 4.125E-0e \\
\hline I & 2.412E-04 & 7.605E-05 & 3.001E-05 & 1.264E-05 & 3.872E-06 \\
\hline $\mathrm{J}$ & 2.375E-04 & 7.455E-05 & 2.934E-05 & 1.194E-05 & 3.800E-06 \\
\hline K & 1.931E-04 & 6.053E-05 & 2.363E-05 & 9.403E-06 & $2.682 \mathrm{E}-0$ \\
\hline L & $2.164 \mathrm{E}-04$ & 7.121E-05 & 2.737E-05 & 1.031E-05 & $3.207 \mathrm{E}-0$ \\
\hline M & 1.964E-04 & 6.156E-05 & 2.262E-05 & 8.778E-06 & $2.569 \mathrm{E}-0$ \\
\hline $\mathrm{N}$ & 1.761E-04 & 5.598E-05 & 1.938E-05 & 7.571E-06 & 2.165E-06 \\
\hline 0 & 2.067E-04 & 6.602E-05 & 2.313E-05 & 9.006E-06 & $2.648 \mathrm{E}-0$ \\
\hline$P$ & 1.534E-04 & 4.778E-05 & 1.737E-05 & 6.679E-06 & 1.811E-0 \\
\hline $\mathrm{Q}$ & 1.963E-04 & 6.081E-05 & 2.053E-05 & 7.460E-06 & $2.088 \mathrm{E}-0$ \\
\hline $\mathrm{R}$ & 2.156E-04 & 6.887E-05 & 2.385E-05 & 9.613E-06 & $2.891 \mathrm{E}-0$ \\
\hline Min & $1.534 E-04$ & 4.778E-05 & 1.737E-05 & 6.679E-06 & $1.811 E-C$ \\
\hline $\operatorname{Max}$ & $2.891 E-04$ & $9.076 E-05$ & $4.459 E-05$ & $1.851 E-05$ & $6.330 E-C$ \\
\hline
\end{tabular}

Table 7.6: Ground-level 95 ${ }^{\text {th }}$ Percentile 0-2 hour Average X/Q $\left(\mathrm{s} / \mathrm{m}^{3}\right)$.

$\begin{array}{cccccc}\text { Site ID } & \mathbf{4 0 0} \mathbf{~ m} & \mathbf{8 0 0} \mathbf{~ m} & \mathbf{1 6 0 0} \mathbf{~ m} & \mathbf{3 2 0 0} \mathbf{~ m} & \mathbf{8 0 0 0} \mathbf{~ m} \\ \text { A } & 2.076 \mathrm{E}-03 & 6.392 \mathrm{E}-04 & 3.295 \mathrm{E}-04 & 1.860 \mathrm{E}-04 & 8.631 \mathrm{E}-05 \\ \text { B } & 3.112 \mathrm{E}-03 & 9.575 \mathrm{E}-04 & 4.946 \mathrm{E}-04 & 2.805 \mathrm{E}-04 & 1.304 \mathrm{E}-04 \\ \text { C } & 3.349 \mathrm{E}-03 & 1.051 \mathrm{E}-03 & 5.197 \mathrm{E}-04 & 2.661 \mathrm{E}-04 & 1.132 \mathrm{E}-04 \\ \text { D } & 2.254 \mathrm{E}-03 & 6.936 \mathrm{E}-04 & 3.582 \mathrm{E}-04 & 2.032 \mathrm{E}-04 & 9.443 \mathrm{E}-05 \\ \text { E } & 2.230 \mathrm{E}-03 & 6.861 \mathrm{E}-04 & 3.544 \mathrm{E}-04 & 2.010 \mathrm{E}-04 & 9.341 \mathrm{E}-05 \\ \text { F } & 6.342 \mathrm{E}-04 & 1.999 \mathrm{E}-04 & 9.791 \mathrm{E}-05 & 4.916 \mathrm{E}-05 & 1.865 \mathrm{E}-05 \\ \text { G } & 1.211 \mathrm{E}-03 & 3.799 \mathrm{E}-04 & 1.891 \mathrm{E}-04 & 1.024 \mathrm{E}-04 & 4.679 \mathrm{E}-05 \\ \text { H } & 9.176 \mathrm{E}-04 & 2.879 \mathrm{E}-04 & 1.425 \mathrm{E}-04 & 7.389 \mathrm{E}-05 & 2.859 \mathrm{E}-05 \\ \text { I } & 1.514 \mathrm{E}-03 & 4.658 \mathrm{E}-04 & 2.379 \mathrm{E}-04 & 1.350 \mathrm{E}-04 & 6.204 \mathrm{E}-05 \\ \text { J } & 1.121 \mathrm{E}-03 & 3.449 \mathrm{E}-04 & 1.909 \mathrm{E}-04 & 1.010 \mathrm{E}-04 & 4.615 \mathrm{E}-05 \\ \text { K } & 9.055 \mathrm{E}-04 & 2.835 \mathrm{E}-04 & 1.409 \mathrm{E}-04 & 7.500 \mathrm{E}-05 & 3.110 \mathrm{E}-05 \\ \text { L } & 2.047 \mathrm{E}-03 & 6.422 \mathrm{E}-04 & 3.176 \mathrm{E}-04 & 1.626 \mathrm{E}-04 & 6.885 \mathrm{E}-05 \\ \text { M } & 8.406 \mathrm{E}-04 & 2.586 \mathrm{E}-04 & 1.403 \mathrm{E}-04 & 7.268 \mathrm{E}-05 & 3.510 \mathrm{E}-05 \\ \text { N } & 1.244 \mathrm{E}-03 & 3.827 \mathrm{E}-04 & 1.977 \mathrm{E}-04 & 1.121 \mathrm{E}-04 & 5.128 \mathrm{E}-05 \\ \text { O } & 1.049 \mathrm{E}-03 & 3.229 \mathrm{E}-04 & 1.467 \mathrm{E}-04 & 8.193 \mathrm{E}-05 & 3.455 \mathrm{E}-05 \\ \text { P } & 7.117 \mathrm{E}-04 & 2.216 \mathrm{E}-04 & 1.043 \mathrm{E}-04 & 5.419 \mathrm{E}-05 & 2.370 \mathrm{E}-05 \\ \text { Q } & 7.644 \mathrm{E}-04 & 2.352 \mathrm{E}-04 & 1.094 \mathrm{E}-04 & 6.110 \mathrm{E}-05 & 2.566 \mathrm{E}-05 \\ \text { R } & 1.818 \mathrm{E}-03 & 5.657 \mathrm{E}-04 & 2.775 \mathrm{E}-04 & 1.428 \mathrm{E}-04 & 5.761 \mathrm{E}-05 \\ \text { Min } & 6.342 \mathrm{E}-04 & 1.999 \mathrm{E}-04 & 9.791 \mathrm{E}-05 & 4.916 \mathrm{E}-05 & 1.865 \mathrm{E}-05 \\ \text { Max } & 3.349 \mathrm{E}-03 & 1.051 \mathrm{E}-03 & 5.197 \mathrm{E}-04 & 2.805 \mathrm{E}-04 & 1.304 \mathrm{E}-04\end{array}$

$\begin{array}{cccccc}\text { Site ID } & \mathbf{4 0 0 ~ \mathbf { ~ }} & \mathbf{8 0 0} \mathbf{~ m} & \mathbf{1 6 0 0} \mathbf{~} & \mathbf{3 2 0 0} \mathbf{~ m} & \mathbf{8 0 0 0} \mathbf{~ m} \\ \text { A } & 8.050 \mathrm{E}-05 & 2.340 \mathrm{E}-05 & 6.990 \mathrm{E}-06 & 2.400 \mathrm{E}-06 & 6.530 \mathrm{E}-07 \\ \text { B } & 1.600 \mathrm{E}-04 & 4.630 \mathrm{E}-05 & 1.380 \mathrm{E}-05 & 4.780 \mathrm{E}-06 & 1.340 \mathrm{E}-06\end{array}$




\begin{tabular}{|c|c|c|c|c|c|}
\hline $\begin{array}{l}\text { Site ID } \\
\text { C }\end{array}$ & $\begin{array}{c}400 \mathrm{~m} \\
1.090 \mathrm{E}-04\end{array}$ & $\begin{array}{c}\mathbf{8 0 0 ~ m} \\
3.200 \mathrm{E}-05\end{array}$ & $\begin{array}{c}1600 \mathrm{~m} \\
9.610 \mathrm{E}-06\end{array}$ & $\begin{array}{c}3200 \mathbf{~ m} \\
3.290 \mathrm{E}-06\end{array}$ & $\begin{array}{c}8000 \mathrm{~m} \\
8.910 \mathrm{E}-07\end{array}$ \\
\hline $\mathrm{D}$ & 8.480 E-05 & $2.470 \mathrm{E}-05$ & 7.410E-06 & $2.530 \mathrm{E}-06$ & $6.850 \mathrm{E}-07$ \\
\hline E & 5.740E-05 & 1.660E-05 & 4.940E-06 & 1.710E-06 & 4.730E-07 \\
\hline $\mathrm{F}$ & $3.150 \mathrm{E}-05$ & $9.250 \mathrm{E}-06$ & 2.800E-06 & 9.390E-07 & $2.450 \mathrm{E}-07$ \\
\hline G & $1.300 \mathrm{E}-04$ & 3.790E-05 & 1.140E-05 & $3.900 \mathrm{E}-06$ & $1.060 \mathrm{E}-06$ \\
\hline $\mathrm{H}$ & 4.330E-05 & $1.280 \mathrm{E}-05$ & 3.870E-06 & 1.310E-06 & $3.430 \mathrm{E}-07$ \\
\hline I & 8.990E-05 & $2.600 \mathrm{E}-05$ & 7.720E-06 & $2.680 \mathrm{E}-06$ & 7.440E-07 \\
\hline $\mathrm{J}$ & $3.520 \mathrm{E}-05$ & $1.020 \mathrm{E}-05$ & $3.050 \mathrm{E}-06$ & $1.040 \mathrm{E}-06$ & 2.820E-07 \\
\hline K & 4.410E-05 & $1.280 \mathrm{E}-05$ & 3.840E-06 & 1.310E-06 & 3.550E-07 \\
\hline $\mathrm{L}$ & $6.150 \mathrm{E}-05$ & $1.780 \mathrm{E}-05$ & 5.330E-06 & $1.820 \mathrm{E}-06$ & $4.890 \mathrm{E}-0$ \\
\hline M & 5.710E-05 & 1.660E-05 & 4.970E-06 & $1.700 \mathrm{E}-06$ & 4.610E-07 \\
\hline $\mathrm{N}$ & 4.960E-05 & 1.440E-05 & $4.280 \mathrm{E}-06$ & $1.480 \mathrm{E}-06$ & 4.110E-07 \\
\hline 0 & $2.870 \mathrm{E}-05$ & 8.340E-06 & $2.500 \mathrm{E}-06$ & $8.560 \mathrm{E}-07$ & 2.330E-07 \\
\hline $\mathrm{P}$ & $2.680 \mathrm{E}-05$ & 7.740E-06 & $1.960 \mathrm{E}-06$ & $6.670 \mathrm{E}-07$ & $1.800 \mathrm{E}-0$ \\
\hline $\mathrm{Q}$ & $2.060 \mathrm{E}-05$ & 6.010E-06 & $1.810 \mathrm{E}-06$ & $6.080 \mathrm{E}-07$ & 1.590E-07 \\
\hline $\mathrm{R}$ & 6.680E-05 & $1.950 \mathrm{E}-05$ & $5.830 \mathrm{E}-06$ & 1.990E-06 & 5.330E-07 \\
\hline Min & $2.060 E-05$ & $6.010 E-06$ & $1.810 E-06$ & $6.080 E-07$ & $1.590 E-0$ \\
\hline Max & $1.600 E-04$ & $4.630 E-05$ & $1.380 E-05$ & $4.780 E-06$ & $1.340 E-C$ \\
\hline
\end{tabular}

\section{Table 7.8: Elevated $50^{\text {th }}$ Percentile $0-2$ hour Average X/Q $\left(\mathrm{s} / \mathrm{m}^{3}\right)$.}

$\begin{array}{cccccc}\text { Site ID } & \mathbf{4 0 0} \mathbf{~ m} & \mathbf{8 0 0} \mathbf{~} & \mathbf{1 6 0 0} \mathbf{~} & \mathbf{3 2 0 0} \mathbf{~} & \mathbf{8 0 0 0} \mathbf{~} \\ \text { A } & 1.044 \mathrm{E}-06 & 1.030 \mathrm{E}-06 & 8.779 \mathrm{E}-07 & 7.833 \mathrm{E}-07 & 5.904 \mathrm{E}-07 \\ \text { B } & 1.057 \mathrm{E}-06 & 1.025 \mathrm{E}-06 & 1.024 \mathrm{E}-06 & 9.237 \mathrm{E}-07 & 6.388 \mathrm{E}-07 \\ \text { C } & 1.723 \mathrm{E}-06 & 1.671 \mathrm{E}-06 & 1.575 \mathrm{E}-06 & 1.532 \mathrm{E}-06 & 9.704 \mathrm{E}-07 \\ \text { D } & 1.648 \mathrm{E}-06 & 1.595 \mathrm{E}-06 & 1.534 \mathrm{E}-06 & 1.477 \mathrm{E}-06 & 9.583 \mathrm{E}-07 \\ \text { E } & 9.322 \mathrm{E}-07 & 8.867 \mathrm{E}-07 & 6.693 \mathrm{E}-07 & 6.405 \mathrm{E}-07 & 4.791 \mathrm{E}-07 \\ \text { F } & 1.865 \mathrm{E}-06 & 1.709 \mathrm{E}-06 & 1.709 \mathrm{E}-06 & 1.479 \mathrm{E}-06 & 9.580 \mathrm{E}-07 \\ \text { G } & 1.497 \mathrm{E}-06 & 1.307 \mathrm{E}-06 & 1.246 \mathrm{E}-06 & 1.234 \mathrm{E}-06 & 7.675 \mathrm{E}-07 \\ \text { H } & 1.534 \mathrm{E}-06 & 1.534 \mathrm{E}-06 & 1.502 \mathrm{E}-06 & 1.478 \mathrm{E}-06 & 9.595 \mathrm{E}-07 \\ \text { I } & 1.285 \mathrm{E}-06 & 1.249 \mathrm{E}-06 & 9.313 \mathrm{E}-07 & 7.075 \mathrm{E}-07 & 4.791 \mathrm{E}-07 \\ \text { J } & 9.715 \mathrm{E}-07 & 9.318 \mathrm{E}-07 & 8.536 \mathrm{E}-07 & 6.793 \mathrm{E}-07 & 4.878 \mathrm{E}-07 \\ \text { K } & 1.490 \mathrm{E}-06 & 1.300 \mathrm{E}-06 & 1.250 \mathrm{E}-06 & 1.164 \mathrm{E}-06 & 7.664 \mathrm{E}-07 \\ \text { L } & 1.249 \mathrm{E}-06 & 1.113 \mathrm{E}-06 & 9.715 \mathrm{E}-07 & 9.369 \mathrm{E}-07 & 4.854 \mathrm{E}-07 \\ \text { M } & 9.914 \mathrm{E}-07 & 9.328 \mathrm{E}-07 & 7.878 \mathrm{E}-07 & 7.385 \mathrm{E}-07 & 4.792 \mathrm{E}-07 \\ \text { N } & 9.568 \mathrm{E}-07 & 9.336 \mathrm{E}-07 & 9.327 \mathrm{E}-07 & 8.545 \mathrm{E}-07 & 4.843 \mathrm{E}-07 \\ \text { O } & 9.709 \mathrm{E}-07 & 9.019 \mathrm{E}-07 & 7.004 \mathrm{E}-07 & 6.401 \mathrm{E}-07 & 4.727 \mathrm{E}-07 \\ \text { P } & 9.493 \mathrm{E}-07 & 9.321 \mathrm{E}-07 & 7.502 \mathrm{E}-07 & 6.535 \mathrm{E}-07 & 4.731 \mathrm{E}-07 \\ \text { Q } & 7.572 \mathrm{E}-07 & 7.464 \mathrm{E}-07 & 6.407 \mathrm{E}-07 & 5.229 \mathrm{E}-07 & 4.015 \mathrm{E}-07 \\ \text { R } & 9.429 \mathrm{E}-07 & 8.764 \mathrm{E}-07 & 7.713 \mathrm{E}-07 & 7.471 \mathrm{E}-07 & 4.715 \mathrm{E}-07 \\ \text { Min } & 7.572 \mathrm{E}-07 & 7.464 \mathrm{E}-07 & 6.407 \mathrm{E}-07 & 5.229 \mathrm{E}-07 & 4.015 \mathrm{E}-07 \\ \text { Max } & 1.865 \mathrm{E}-06 & 1.709 \mathrm{E}-06 & 1.709 \mathrm{E}-06 & 1.532 \mathrm{E}-06 & 9.704 \mathrm{E}-07\end{array}$


Table 7.9: Elevated $95^{\text {th }}$ Percentile 0-2 hour Average X/Q $\left(\mathrm{s} / \mathrm{m}^{3}\right)$.

$\begin{array}{cccccc}\text { Site ID } & \mathbf{4 0 0} \mathbf{~ m} & \mathbf{8 0 0} \mathbf{~} & \mathbf{1 6 0 0} \mathbf{~} & \mathbf{3 2 0 0} \mathbf{~} & \mathbf{8 0 0 0} \mathbf{~} \\ \text { A } & 5.676 \mathrm{E}-06 & 4.279 \mathrm{E}-06 & 3.750 \mathrm{E}-06 & 2.599 \mathrm{E}-06 & 1.758 \mathrm{E}-06 \\ \text { B } & 4.543 \mathrm{E}-06 & 4.258 \mathrm{E}-06 & 3.920 \mathrm{E}-06 & 3.695 \mathrm{E}-06 & 1.970 \mathrm{E}-06 \\ \text { C } & 5.319 \mathrm{E}-06 & 5.319 \mathrm{E}-06 & 5.315 \mathrm{E}-06 & 5.242 \mathrm{E}-06 & 3.914 \mathrm{E}-06 \\ \text { D } & 4.972 \mathrm{E}-06 & 4.972 \mathrm{E}-06 & 4.972 \mathrm{E}-06 & 4.928 \mathrm{E}-06 & 2.598 \mathrm{E}-06 \\ \text { E } & 5.683 \mathrm{E}-06 & 3.272 \mathrm{E}-06 & 2.495 \mathrm{E}-06 & 2.298 \mathrm{E}-06 & 1.278 \mathrm{E}-06 \\ \text { F } & 5.712 \mathrm{E}-06 & 4.973 \mathrm{E}-06 & 4.973 \mathrm{E}-06 & 3.724 \mathrm{E}-06 & 2.556 \mathrm{E}-06 \\ \text { G } & 5.289 \mathrm{E}-06 & 4.990 \mathrm{E}-06 & 4.662 \mathrm{E}-06 & 3.913 \mathrm{E}-06 & 3.148 \mathrm{E}-06 \\ \text { H } & 5.145 \mathrm{E}-06 & 5.123 \mathrm{E}-06 & 5.123 \mathrm{E}-06 & 5.123 \mathrm{E}-06 & 3.833 \mathrm{E}-06 \\ \text { I } & 6.165 \mathrm{E}-06 & 4.624 \mathrm{E}-06 & 3.919 \mathrm{E}-06 & 3.351 \mathrm{E}-06 & 1.940 \mathrm{E}-06 \\ \text { J } & 4.967 \mathrm{E}-06 & 3.791 \mathrm{E}-06 & 2.708 \mathrm{E}-06 & 2.513 \mathrm{E}-06 & 1.568 \mathrm{E}-06 \\ \text { K } & 5.119 \mathrm{E}-06 & 4.104 \mathrm{E}-06 & 3.733 \mathrm{E}-06 & 3.534 \mathrm{E}-06 & 1.940 \mathrm{E}-06 \\ \text { L } & 4.995 \mathrm{E}-06 & 4.995 \mathrm{E}-06 & 4.995 \mathrm{E}-06 & 3.506 \mathrm{E}-06 & 2.559 \mathrm{E}-06 \\ \text { M } & 4.797 \mathrm{E}-06 & 3.731 \mathrm{E}-06 & 2.658 \mathrm{E}-06 & 2.465 \mathrm{E}-06 & 1.290 \mathrm{E}-06 \\ \text { N } & 3.731 \mathrm{E}-06 & 3.273 \mathrm{E}-06 & 2.490 \mathrm{E}-06 & 2.465 \mathrm{E}-06 & 1.285 \mathrm{E}-06 \\ \text { O } & 4.597 \mathrm{E}-06 & 2.598 \mathrm{E}-06 & 2.399 \mathrm{E}-06 & 1.906 \mathrm{E}-06 & 1.282 \mathrm{E}-06 \\ \text { P } & 5.700 \mathrm{E}-06 & 3.725 \mathrm{E}-06 & 2.486 \mathrm{E}-06 & 1.960 \mathrm{E}-06 & 1.278 \mathrm{E}-06 \\ \text { Q } & 3.821 \mathrm{E}-06 & 2.225 \mathrm{E}-06 & 1.799 \mathrm{E}-06 & 1.479 \mathrm{E}-06 & 9.583 \mathrm{E}-07 \\ \text { R } & 4.544 \mathrm{E}-06 & 3.309 \mathrm{E}-06 & 2.541 \mathrm{E}-06 & 2.490 \mathrm{E}-06 & 1.556 \mathrm{E}-06 \\ \text { Min } & 3.731 \mathrm{E}-06 & 2.225 \mathrm{E}-06 & 1.799 \mathrm{E}-06 & 1.479 \mathrm{E}-06 & 9.583 \mathrm{E}-07 \\ \text { Max } & 6.165 \mathrm{E}-06 & 5.319 \mathrm{E}-06 & 5.315 \mathrm{E}-06 & 5.242 \mathrm{E}-06 & 3.914 \mathrm{E}-06\end{array}$

Table 7.10: Elevated Annual Average X/Q $\left(\mathrm{s} / \mathrm{m}^{3}\right)$.

$\begin{array}{cccccc}\text { Site ID } & \mathbf{4 0 0} \mathbf{~ m} & \mathbf{8 0 0} \mathbf{~ m} & \mathbf{1 6 0 0} \mathbf{~} & \mathbf{3 2 0 0} \mathbf{~} & \mathbf{8 0 0 0} \mathbf{~} \\ \text { A } & 8.700 \mathrm{E}-08 & 5.920 \mathrm{E}-08 & 4.460 \mathrm{E}-08 & 4.360 \mathrm{E}-08 & 2.410 \mathrm{E}-08 \\ \text { B } & 3.570 \mathrm{E}-08 & 4.340 \mathrm{E}-08 & 5.030 \mathrm{E}-08 & 4.970 \mathrm{E}-08 & 2.840 \mathrm{E}-08 \\ \text { C } & 1.810 \mathrm{E}-08 & 3.930 \mathrm{E}-08 & 9.490 \mathrm{E}-08 & 1.310 \mathrm{E}-07 & 7.950 \mathrm{E}-08 \\ \text { D } & 1.580 \mathrm{E}-08 & 2.630 \mathrm{E}-08 & 8.340 \mathrm{E}-08 & 1.280 \mathrm{E}-07 & 8.220 \mathrm{E}-08 \\ \text { E } & 1.440 \mathrm{E}-07 & 5.560 \mathrm{E}-08 & 2.680 \mathrm{E}-08 & 2.920 \mathrm{E}-08 & 1.650 \mathrm{E}-08 \\ \text { F } & 2.170 \mathrm{E}-07 & 1.290 \mathrm{E}-07 & 8.860 \mathrm{E}-08 & 7.610 \mathrm{E}-08 & 4.590 \mathrm{E}-08 \\ \text { G } & 1.300 \mathrm{E}-07 & 6.980 \mathrm{E}-08 & 5.630 \mathrm{E}-08 & 6.480 \mathrm{E}-08 & 4.850 \mathrm{E}-08 \\ \text { H } & 3.110 \mathrm{E}-08 & 3.100 \mathrm{E}-08 & 7.860 \mathrm{E}-08 & 1.270 \mathrm{E}-07 & 8.390 \mathrm{E}-08 \\ \text { I } & 1.190 \mathrm{E}-07 & 7.430 \mathrm{E}-08 & 4.900 \mathrm{E}-08 & 4.290 \mathrm{E}-08 & 2.280 \mathrm{E}-08 \\ \text { J } & 7.420 \mathrm{E}-08 & 4.450 \mathrm{E}-08 & 3.200 \mathrm{E}-08 & 3.180 \mathrm{E}-08 & 1.800 \mathrm{E}-08 \\ \text { K } & 8.370 \mathrm{E}-08 & 4.590 \mathrm{E}-08 & 4.790 \mathrm{E}-08 & 5.990 \mathrm{E}-08 & 3.090 \mathrm{E}-08 \\ \text { L } & 2.180 \mathrm{E}-08 & 1.820 \mathrm{E}-08 & 5.650 \mathrm{E}-08 & 7.890 \mathrm{E}-08 & 4.130 \mathrm{E}-08 \\ \text { M } & 1.120 \mathrm{E}-07 & 5.980 \mathrm{E}-08 & 4.420 \mathrm{E}-08 & 3.910 \mathrm{E}-08 & 1.830 \mathrm{E}-08 \\ \text { N } & 4.740 \mathrm{E}-08 & 4.850 \mathrm{E}-08 & 4.230 \mathrm{E}-08 & 3.940 \mathrm{E}-08 & 2.180 \mathrm{E}-08 \\ \text { O } & 1.290 \mathrm{E}-07 & 4.890 \mathrm{E}-08 & 2.510 \mathrm{E}-08 & 2.750 \mathrm{E}-08 & 1.600 \mathrm{E}-08 \\ \text { P } & 1.340 \mathrm{E}-07 & 6.290 \mathrm{E}-08 & 3.280 \mathrm{E}-08 & 3.080 \mathrm{E}-08 & 1.810 \mathrm{E}-08 \\ \text { Q } & 2.400 \mathrm{E}-07 & 8.950 \mathrm{E}-08 & 3.370 \mathrm{E}-08 & 3.780 \mathrm{E}-08 & 2.490 \mathrm{E}-08 \\ \text { R } & 1.710 \mathrm{E}-07 & 9.650 \mathrm{E}-08 & 6.280 \mathrm{E}-08 & 5.540 \mathrm{E}-08 & 3.060 \mathrm{E}-08 \\ \text { Min } & 1.580 \mathrm{E}-08 & 1.820 \mathrm{E}-08 & 2.510 \mathrm{E}-08 & 2.750 \mathrm{E}-08 & 1.600 \mathrm{E}-08 \\ \text { Max } & 2.400 \mathrm{E}-07 & 1.290 \mathrm{E}-07 & 9.490 \mathrm{E}-08 & 1.310 \mathrm{E}-07 & 8.390 \mathrm{E}-08\end{array}$


PNNL-19217

Table 7.11: Ground-level $50^{\text {th }}$ Percentile X/Q's $\left(\mathrm{s} / \mathrm{m}^{3}\right)$ for Intermediate Averaging Times at $400 \mathrm{~m}$.

\begin{tabular}{|c|c|c|c|c|c|c|}
\hline Site ID & $0-2$ hour & $0-8 \mathrm{hr}$ & $8-24 \mathrm{hr}$ & 1-4day & 4-30day & Annual \\
\hline A & $2.859 \mathrm{E}-04$ & $2.319 \mathrm{E}-04$ & $2.088 \mathrm{E}-04$ & $1.663 \mathrm{E}-04$ & $1.200 \mathrm{E}-04$ & 8.050E-05 \\
\hline B & 2.891E-04 & 2.622E-04 & 2.496E-04 & 2.245E-04 & 1.928E-04 & $1.600 \mathrm{E}-04$ \\
\hline C & $2.845 \mathrm{E}-04$ & $2.428 \mathrm{E}-04$ & 2.243E-04 & $1.888 \mathrm{E}-04$ & $1.475 \mathrm{E}-04$ & 1.090E-04 \\
\hline$D$ & $2.535 \mathrm{E}-04$ & 2.115E-04 & 1.932E-04 & $1.588 \mathrm{E}-04$ & 1.197E-04 & 8.480E-05 \\
\hline$E$ & 2.349E-04 & 1.861E-04 & 1.656E-04 & $1.286 \mathrm{E}-04$ & 8.948E-05 & 5.740E-05 \\
\hline$F$ & $2.110 \mathrm{E}-04$ & 1.541E-04 & 1.317E-04 & 9.360E-05 & 5.735E-05 & $3.150 \mathrm{E}-05$ \\
\hline G & $2.423 E-04$ & 2.186E-04 & $2.076 \mathrm{E}-04$ & $1.857 \mathrm{E}-04$ & $1.582 \mathrm{E}-04$ & $1.300 \mathrm{E}-04$ \\
\hline $\mathrm{H}$ & $2.373 \mathrm{E}-04$ & $1.791 \mathrm{E}-04$ & $1.556 \mathrm{E}-04$ & $1.147 \mathrm{E}-04$ & 7.401E-05 & 4.330E-05 \\
\hline I & $2.412 \mathrm{E}-04$ & 2.049E-04 & 1.888E-04 & $1.582 \mathrm{E}-04$ & 1.227E-04 & 8.990E-05 \\
\hline $\mathrm{J}$ & 2.375E-04 & $1.732 \mathrm{E}-04$ & 1.479E-04 & $1.050 \mathrm{E}-04$ & 6.424E-05 & $3.520 \mathrm{E}-05$ \\
\hline K & 1.931E-04 & $1.513 \mathrm{E}-04$ & 1.339E-04 & $1.027 \mathrm{E}-04$ & 7.023E-05 & 4.410E-05 \\
\hline $\mathrm{L}$ & 2.164E-04 & $1.758 \mathrm{E}-04$ & 1.584E-04 & $1.264 \mathrm{E}-04$ & 9.142E-05 & $6.150 \mathrm{E}-05$ \\
\hline$M$ & 1.964E-04 & $1.601 \mathrm{E}-04$ & 1.446E-04 & $1.158 \mathrm{E}-04$ & 8.427E-05 & $5.710 \mathrm{E}-05$ \\
\hline $\mathrm{N}$ & $1.761 \mathrm{E}-04$ & $1.428 \mathrm{E}-04$ & $1.286 \mathrm{E}-04$ & $1.025 \mathrm{E}-04$ & 7.394E-05 & 4.960E-05 \\
\hline $\mathrm{O}$ & 2.067E-04 & 1.491E-04 & 1.267E-04 & 8.890E-05 & 5.346E-05 & $2.870 \mathrm{E}-05$ \\
\hline $\mathrm{P}$ & 1.534E-04 & 1.150E-04 & 9.952E-05 & 7.278E-05 & 4.644E-05 & 2.680E-05 \\
\hline Q & $1.963 \mathrm{E}-04$ & $1.352 \mathrm{E}-04$ & 1.122E-04 & 7.490E-05 & 4.191E-05 & $2.060 \mathrm{E}-05$ \\
\hline $\mathrm{R}$ & 2.156E-04 & $1.776 \mathrm{E}-04$ & 1.612E-04 & 1.307E-04 & 9.663E-05 & 6.680E-05 \\
\hline Min & $1.534 E-04$ & $1.150 E-04$ & 9.952E-05 & $7.278 E-05$ & 4.191E-05 & $2.060 E-05$ \\
\hline Max & $2.891 E-04$ & $2.622 E-04$ & $2.496 E-04$ & $2.245 E-04$ & $1.928 E-04$ & $1.600 E-04$ \\
\hline
\end{tabular}

Table 7.12: Ground-level $95^{\text {th }}$ Percentile $X / Q$ 's $\left(\mathrm{s} / \mathrm{m}^{3}\right)$ for Intermediate Averaging Times at $400 \mathrm{~m}$.

\begin{tabular}{|c|c|c|c|c|c|c|}
\hline Site ID & 0-2 hour & $0-8 \mathrm{hr}$ & $8-24 \mathrm{hr}$ & 1-4day & 4-30day & Annual \\
\hline A & $2.076 \mathrm{E}-03$ & $1.213 \mathrm{E}-03$ & $9.272 \mathrm{E}-04$ & $5.176 \mathrm{E}-04$ & $2.241 \mathrm{E}-04$ & 8.050E-05 \\
\hline B & 3.112E-03 & $1.905 \mathrm{E}-03$ & 1.491E-03 & 8.753E-04 & 4.076E-04 & $1.600 \mathrm{E}-04$ \\
\hline C & 3.349E-03 & 1.901E-03 & 1.432E-03 & 7.748E-04 & 3.207E-04 & 1.090E-04 \\
\hline D & $2.254 \mathrm{E}-03$ & $1.310 \mathrm{E}-03$ & 9.992E-04 & 5.548E-04 & $2.384 \mathrm{E}-04$ & 8.480E-05 \\
\hline$E$ & $2.230 \mathrm{E}-03$ & $1.218 \mathrm{E}-03$ & 8.998E-04 & 4.667E-04 & 1.818E-04 & $5.740 \mathrm{E}-05$ \\
\hline$F$ & $6.342 \mathrm{E}-04$ & 3.861E-04 & 3.012E-04 & $1.758 \mathrm{E}-04$ & $8.112 \mathrm{E}-05$ & 3.150E-05 \\
\hline G & $1.211 \mathrm{E}-03$ & 8.373E-04 & 6.963E-04 & 4.666E-04 & $2.626 \mathrm{E}-04$ & $1.300 \mathrm{E}-04$ \\
\hline $\mathrm{H}$ & $9.176 \mathrm{E}-04$ & 5.539E-04 & 4.303E-04 & 2.488E-04 & $1.133 \mathrm{E}-04$ & 4.330E-05 \\
\hline 1 & $1.514 \mathrm{E}-03$ & 9.492E-04 & 7.516E-04 & $4.529 \mathrm{E}-04$ & 2.189E-04 & 8.990E-05 \\
\hline $\mathrm{J}$ & $1.121 \mathrm{E}-03$ & 6.326E-04 & 4.752E-04 & $2.554 \mathrm{E}-04$ & $1.047 \mathrm{E}-04$ & $3.520 \mathrm{E}-05$ \\
\hline K & $9.055 \mathrm{E}-04$ & 5.494E-04 & 4.280E-04 & 2.489E-04 & $1.143 \mathrm{E}-04$ & 4.410E-05 \\
\hline L & $2.047 \mathrm{E}-03$ & $1.147 \mathrm{E}-03$ & 8.582E-04 & 4.577E-04 & $1.856 \mathrm{E}-04$ & $6.150 \mathrm{E}-05$ \\
\hline$M$ & 8.406E-04 & 5.389E-04 & 4.315E-04 & 2.663E-04 & $1.332 \mathrm{E}-04$ & $5.710 \mathrm{E}-05$ \\
\hline $\mathrm{N}$ & 1.244E-03 & 7.302E-04 & 5.595E-04 & 3.139E-04 & 1.369E-04 & 4.960E-05 \\
\hline $\mathrm{O}$ & $1.049 \mathrm{E}-03$ & 5.786E-04 & 4.297E-04 & 2.253E-04 & 8.919E-05 & 2.870E-05 \\
\hline $\mathrm{P}$ & 7.117E-04 & 4.138E-04 & 3.156E-04 & $1.752 \mathrm{E}-04$ & 7.531E-05 & $2.680 \mathrm{E}-05$ \\
\hline Q & 7.644E-04 & 4.206E-04 & 3.120E-04 & $1.631 \mathrm{E}-04$ & $6.432 \mathrm{E}-05$ & 2.060E-05 \\
\hline $\mathrm{R}$ & $1.818 \mathrm{E}-03$ & $1.053 \mathrm{E}-03$ & 8.012E-04 & 4.430E-04 & $1.892 \mathrm{E}-04$ & 6.680E-05 \\
\hline Min & $6.342 E-04$ & $3.861 E-04$ & $3.012 E-04$ & $1.631 E-04$ & $6.432 E-05$ & $2.060 E-05$ \\
\hline Max & $3.349 E-03$ & $1.905 E-03$ & 1.491E-03 & 8.753E-04 & $4.076 E-04$ & $1.600 E-04$ \\
\hline
\end{tabular}


PNNL-19217

Table 7.13: Elevated $50^{\text {th }}$ Percentile X/Q's $\left(\mathrm{s} / \mathrm{m}^{3}\right)$ for Intermediate Averaging Times at 400 $\mathrm{m}$.

\begin{tabular}{|c|c|c|c|c|c|c|}
\hline Site ID & $0-2$ hour & $0-8 \mathrm{hr}$ & $8-24 h r$ & 1-4day & 4-30day & Annual \\
\hline A & 1.044E-06 & $6.923 \mathrm{E}-07$ & 5.637E-07 & $3.610 \mathrm{E}-07$ & $1.903 \mathrm{E}-07$ & 8.700E-08 \\
\hline B & 1.057E-06 & $6.037 \mathrm{E}-07$ & 4.562E-07 & 2.484E-07 & $1.038 \mathrm{E}-07$ & 3.570E-08 \\
\hline C & $1.723 \mathrm{E}-06$ & 8.112E-07 & 5.567E-07 & 2.458E-07 & 7.605E-08 & $1.810 \mathrm{E}-08$ \\
\hline D & $1.648 \mathrm{E}-06$ & $7.643 \mathrm{E}-07$ & 5.205E-07 & $2.261 \mathrm{E}-07$ & 6.832E-08 & 1.580E-08 \\
\hline$E$ & $9.322 \mathrm{E}-07$ & 6.845E-07 & 5.866E-07 & 4.196E-07 & $2.594 \mathrm{E}-07$ & $1.440 \mathrm{E}-07$ \\
\hline $\mathrm{F}$ & 1.865E-06 & 1.307E-06 & 1.094E-06 & 7.437E-07 & 4.274E-07 & 2.170E-07 \\
\hline G & 1.497E-06 & $9.994 \mathrm{E}-07$ & 8.166E-07 & $5.268 \mathrm{E}-07$ & $2.808 \mathrm{E}-07$ & 1.300E-07 \\
\hline $\mathrm{H}$ & $1.534 \mathrm{E}-06$ & 8.052E-07 & 5.834E-07 & 2.899E-07 & $1.062 \mathrm{E}-07$ & 3.110E-08 \\
\hline I & $1.285 \mathrm{E}-06$ & 8.671E-07 & 7.122E-07 & 4.648E-07 & $2.518 \mathrm{E}-07$ & 1.190E-07 \\
\hline $\mathrm{J}$ & 9.715E-07 & 6.350E-07 & 5.134E-07 & 3.236E-07 & 1.669E-07 & 7.420E-08 \\
\hline K & $1.490 \mathrm{E}-06$ & $9.256 \mathrm{E}-07$ & 7.296E-07 & 4.353E-07 & $2.074 \mathrm{E}-07$ & 8.370E-08 \\
\hline L & $1.249 \mathrm{E}-06$ & 6.396E-07 & 4.577E-07 & $2.214 \mathrm{E}-07$ & 7.805E-08 & 2.180E-08 \\
\hline$M$ & 9.914E-07 & 6.913E-07 & 5.773E-07 & 3.904E-07 & $2.226 \mathrm{E}-07$ & 1.120E-07 \\
\hline $\mathrm{N}$ & $9.568 \mathrm{E}-07$ & $5.822 \mathrm{E}-07$ & 4.541E-07 & 2.649E-07 & $1.222 \mathrm{E}-07$ & 4.740E-08 \\
\hline $\mathrm{O}$ & $9.709 \mathrm{E}-07$ & $6.954 \mathrm{E}-07$ & 5.885E-07 & 4.098E-07 & $2.437 \mathrm{E}-07$ & $1.290 \mathrm{E}-07$ \\
\hline $\mathrm{P}$ & 9.493E-07 & 6.868E-07 & 5.842E-07 & 4.112E-07 & $2.483 \mathrm{E}-07$ & 1.340E-07 \\
\hline Q & $7.572 \mathrm{E}-07$ & $6.262 \mathrm{E}-07$ & 5.695E-07 & 4.634E-07 & $3.447 \mathrm{E}-07$ & $2.400 \mathrm{E}-07$ \\
\hline $\mathrm{R}$ & $9.429 \mathrm{E}-07$ & 7.110E-07 & $6.174 \mathrm{E}-07$ & 4.545E-07 & $2.928 \mathrm{E}-07$ & $1.710 \mathrm{E}-07$ \\
\hline Min & $7.572 E-07$ & $5.822 E-07$ & $4.541 E-07$ & $2.214 E-07$ & $6.832 E-08$ & $1.580 E-08$ \\
\hline $\operatorname{Max}$ & $1.865 E-06$ & $1.307 E-06$ & $1.094 E-06$ & 7.437E-07 & $4.274 E-07$ & $2.400 E-07$ \\
\hline
\end{tabular}

Table 7.14: Elevated $95^{\text {th }}$ Percentile X/Q's $\left(\mathrm{s}^{\mathrm{m}} \mathrm{m}^{3}\right)$ for Intermediate Averaging Times at 400 $\mathrm{m}$.

\begin{tabular}{|c|c|c|c|c|c|c|}
\hline Site ID & 0-2 hour & $0-8 \mathrm{hr}$ & $8-24 \mathrm{hr}$ & 1-4day & 4-30day & Annual \\
\hline A & $5.676 \mathrm{E}-06$ & $2.845 \mathrm{E}-06$ & $2.014 \mathrm{E}-06$ & $9.518 \mathrm{E}-07$ & 3.245E-07 & $8.700 \mathrm{E}-08$ \\
\hline B & 4.543E-06 & 2.039E-06 & 1.366E-06 & 5.726E-07 & 1.644E-07 & 3.570E-08 \\
\hline C & 5.319E-06 & $2.079 \mathrm{E}-06$ & 1.299E-06 & 4.688E-07 & 1.085E-07 & 1.810E-08 \\
\hline$D$ & 4.972E-06 & 1.921E-06 & 1.194E-06 & 4.256E-07 & 9.675E-08 & 1.580E-08 \\
\hline$E$ & 5.683E-06 & 3.095E-06 & $2.284 \mathrm{E}-06$ & 1.181E-06 & 4.584E-07 & 1.440E-07 \\
\hline $\mathrm{F}$ & 5.712E-06 & 3.326E-06 & 2.538E-06 & 1.412E-06 & 6.081E-07 & 2.170E-07 \\
\hline G & 5.289E-06 & $2.866 \mathrm{E}-06$ & $2.110 \mathrm{E}-06$ & 1.085E-06 & 4.179E-07 & $1.300 \mathrm{E}-07$ \\
\hline $\mathrm{H}$ & 5.145E-06 & 2.211E-06 & 1.449E-06 & 5.797E-07 & $1.555 \mathrm{E}-07$ & 3.110E-08 \\
\hline I & 6.165E-06 & 3.210E-06 & 2.316E-06 & 1.141E-06 & 4.128E-07 & 1.190E-07 \\
\hline $\mathrm{J}$ & 4.967E-06 & $2.479 \mathrm{E}-06$ & 1.751E-06 & 8.238E-07 & 2.790E-07 & 7.420E-08 \\
\hline K & 5.119E-06 & 2.593E-06 & 1.846E-06 & 8.824E-07 & 3.059E-07 & 8.370E-08 \\
\hline $\mathrm{L}$ & 4.995E-06 & $2.034 \mathrm{E}-06$ & $1.298 \mathrm{E}-06$ & 4.896E-07 & 1.208E-07 & 2.180E-08 \\
\hline$M$ & 4.797E-06 & 2.577E-06 & 1.889E-06 & 9.629E-07 & 3.659E-07 & 1.120E-07 \\
\hline $\mathrm{N}$ & 3.731E-06 & 1.813E-06 & 1.264E-06 & 5.774E-07 & 1.876E-07 & 4.740E-08 \\
\hline $\mathrm{O}$ & 4.597E-06 & $2.546 \mathrm{E}-06$ & 1.895E-06 & 9.982E-07 & 3.977E-07 & 1.290 E-07 \\
\hline $\mathrm{P}$ & $5.700 \mathrm{E}-06$ & $3.066 \mathrm{E}-06$ & 2.249E-06 & 1.148E-06 & 4.368E-07 & $1.340 \mathrm{E}-07$ \\
\hline Q & 3.821E-06 & 2.418E-06 & 1.923E-06 & 1.171E-06 & 5.740E-07 & $2.400 \mathrm{E}-07$ \\
\hline $\mathrm{R}$ & 4.544E-06 & 2.642E-06 & 2.015E-06 & 1.119E-06 & 4.806E-07 & 1.710E-07 \\
\hline Min & $3.731 E-06$ & $1.813 E-06$ & $1.194 E-06$ & 4.256E-07 & $9.675 E-08$ & $1.580 E-08$ \\
\hline $\operatorname{Max}$ & $6.165 E-06$ & 3.326E-06 & $2.538 E-06$ & $1.412 E-06$ & $6.081 E-07$ & $2.400 E-07$ \\
\hline
\end{tabular}




\subsection{RECOMMENDATIONS}

Following the guidance of RG 1.145 (NRC 1983), PAVAN calculates X/Q at various percentiles for the 0-2 hour averaging period as well as an overall site annual average. The 50th and 95th percentiles form the basis of environmental and safety evaluations, respectively. Use of the provided dispersion parameters to estimate radioactive material dispersion is appropriate for a generic site based on the representative meteorology from 18 different nuclear sites. 


\subsection{REFERENCES}

10 CFR Part 52. Code of Federal Regulations, Title 10, Energy, Part 52, "Early Site Permits; Standard Design Certifications, and Combined Licenses for Nuclear Power Plants."

10 CFR Part 100. Code of Federal Regulations, Title 10, Energy, Part 100, "Reactor Site Criteria."

G. A. Briggs, "Plume Rise,' AEC Critical Review Series, TID-25075, 1969.

NUREG/CR-2858, PLN-4413, "PAVAN: An Atmospheric Dispersion Program for Evaluating Design Basis Accidental Releases of Radioactive Materials from Nuclear Power Stations". T. J. Bander, November 1982.

U.S. Nuclear Regulatory Commission (NRC). 2007. Meteorological Monitoring Programs for Nuclear Power Plants. Regulatory Guide 1.23, Rev. 1, NRC, Washington, D.C.

U.S. Nuclear Regulatory Commission (NRC). 1983. Atmospheric Dispersion Models for Evaluating Design Basis Accidents at Nuclear Power Plants. Regulatory Guide 1.145, Revision 1, Washington D.C.

U.S. Nuclear Regulatory Commission (NRC). 1977. Methods for Estimating Atmospheric Transport and Dispersion of Gaseous Effluents in Routine Releases from Light-Water-Cooled Reactors. Regulatory Guide 1.111, Rev. 1, NRC, Washington, D.C. 
PNNL-19217 


\section{Appendix A - PAVAN Input/Output Files (Provided Electronically)}




\section{Appendix B - Appendix B QA Record (Provided Electronically)}


PNNL-19217 



\section{Distribution}

No. of

Copies

\#

\# Local Distribution

Pacific Northwest National Laboratory

Peter Lowry

Jeremy Rishel

Steven Ross
Mailstop K6-52

MailstopK9-30

Mailstop K6-52
No. of

Copies

Distr.1 


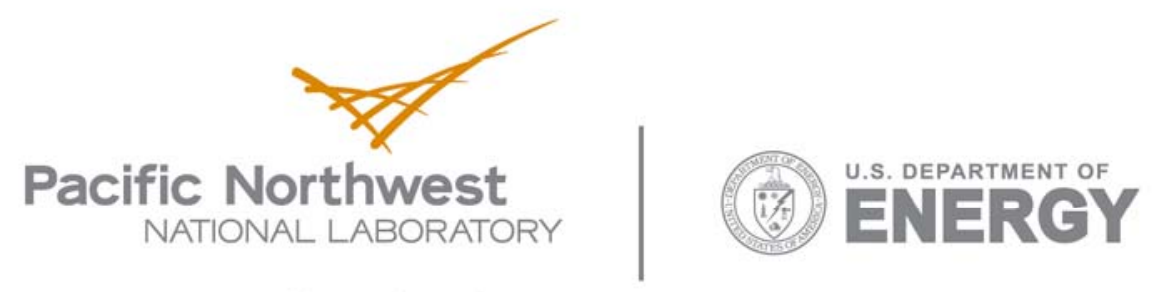

902 Battelle Boulevard

P.O. Box 999

Richland, WA 99352

1-888-375-PNNL (7665)

www.pnl.gov 